\title{
L'OBIEZIONE DI COSCIENZA AL GIURAMENTO NELLA GIURISPRUDENZA DELLA CORTE COSTITUZIONALE DELLA REPUBBLICA ITALIANA
}

DOI: http://dx.doi.org/10.12775/TSP-W.2020.008

Conscientious objection regarding an oath in the judicature of the Constitutional Court of the Italian Republic (Summary) The article raises the issue of conscientious objection regarding an oath in the Italian legal system. Decisions of the Constitutional Court of the Italian Republic show this objection in the light of the norms of criminal and civil procedure codes containing terms and phrases relating to God and religious values, which were questioned and changed in the amended codes for the obligation to tell the truth. Conscientious objection regarding an oath occurs when the swearer's conscience contradicts the law and does not allow him/her to comply with the given legal norm unless the manifestation of disobedience has been legalized or legally justified and remains unpunished in both cases.

Keywords: conscientious objection, Constitutional Court of the Italian Republic, oath, judicature, constitution

Sprzeciw sumienia wobec przysięgi w orzecznictwie Trybunału Konstytucyjnego Republiki Włoskiej. Artykuł ukazuje zagadnienie sprzeciwu sumienia odnośnie do przysięgi we włoskim systemie prawnym. Orzeczenia Trybunału Konstytucyjnego Republiki Włoskiej ukazują tenże sprzeciw w świetle norm kodeksów postępowania karnego i postępowania cywilnego zawierających formuły odnoszące się do Boga i do

${ }^{1}$ Dr Piotr Lewandowski - Department of Practical Theology, Faculty of Theology, The Adam Mickiewicz University in Poznań, ORCID 0000-0002-0600-004X, e-mail: piolew1@ amu. edu.pl. 
wartości religijnych, które zakwestionowano i zmieniono w znowelizowanych kodeksach na obowiązek mówienia prawdy. Sprzeciw sumienia względem przysięgi zachodzi wtedy, gdy sumienie przysięgającego sprzeciwia się nakazom prawa i nie pozwala mu na zachowanie danej normy prawnej, chyba że przejaw nieposłuszeństwa został zalegalizowany lub prawnie uzasadniony, pozostając w obu przypadkach bezkarnym.

Słowa kluczowe: sprzeciw sumienia, Trybunał Konstytucyjny Republiki Włoskiej, przysięga, orzecznictwo,

\section{PREMESSA}

La libertà relativa alle proprie convinzioni, la libertà di coscienza e quella che permette di far propria una determinata concezione del mondo, nella sua dimensione interiore, è un diritto assoluto che non può essere soggetto a limitazione $^{2}$.

La coscienza, intima essenza del soggetto, formata e costituita da valori spirituali, etici, soggettivamente consapevoli e inderogabili unita al valore insostituibile della vita della persona umana si colloca alla base del rifiuto di obbedire a un ordine, a una prescrizione giuridica ritenuta fortemente contrastante con la propria scelta ${ }^{3}$.

In diversi settori ed attività umane vi sono persone sottoposte a situazioni che vanno a determinare conflitti con la propria coscienza e spingono ad agire in conformità al diritto suggerito dalla coscienza stessa nell'esercizio della libertà dalla coercizione contro la propria coscienza ${ }^{4}$.

Il conflitto che maggiormente rappresenta l'essenza di scelte interiori e coinvolge la coscienza riguarda il giuramento che l'individuo è tenuto a prestare in specifiche circostanze previste dalla legge 5 .

L'oggetto di questa elaborazione è l' analisi dell'obiezione al giuramento nelle sentenze e nelle ordinanze della Corte costituzionale della Repubblica Italiana. La Corte ha giudicato la legittimità costituzionale del dovere del testimone di prestare giuramento nel processo civile quanto in quello penale. La Corte costituzionale ha dichiarato che la formula del giuramento deve essere modificata

${ }^{2}$ Cfr. A. Zoll, Klauzula sumienia, in P. Stanisz, J. Pawlikowski, M. Ordon (a cura di), Sprzeciw sumienia w praktyce medycznej-aspekty etyczne i prawne, Lublin 2014, p. 79.

${ }^{3}$ Cfr. G. Dammacco, L'obiezione di coscienza nella giurisprudenza della Corte costituzionale, in R. Botta (a cura di), Diritto ecclesiastico e Corte costituzionale, Napoli 2006, p. 116.

${ }^{4}$ Cfr. M. Skwarczyński, Sprzeciw sumienia w europejskim i krajowym systemie ochrony praw człowieka, ,Przegląd Sejmowy”, 6 (119)/2013, p. 10.

${ }^{5}$ Cfr. G. Dammacco, op.cit., p. 124. 
a causa dell'obiezione di coscienza di coloro i quali intendono negare il riferimento a Dio, sostituendola con un impegno a dire tutta la verità senza riferimenti religiosi.

\section{NOZIONE DELL'OBIEZIONE DI COSCIENZA}

L'obiezione di coscienza, concetto di difficile definizione è un fenomeno individuale che rientra nei suoi aspetti ideali, teorici e pratici, alle convinzioni personali che guidano la vita e alla quale vanno conferite un senso e un significato. La possibilità che una opzione personale, relativa alla sfera più profonda e impenetrabile dell'essenza umana possa divenire rilevante in ambito sociale deriva dalla possibilità di condivisione e dalla consideratezza diffusa del senso comune, che non sempre include internamente i suoi elementi concreti qualitativi ${ }^{6}$.

È un esercizio tramite il quale un individuo può decidere di assumere un dato comportamento contrapposto rispetto a determinate norme giuridiche ma legittimato dall'ordinamento al fine di tutelare le posizioni e convinzioni morali e religiose ${ }^{7}$.

L'obiezione di coscienza è tradizionalmente definita come il rifiuto individuale di sottostare a una legge dell' autorità, ad una norma giuridica, espresso dalla presenza nella propria coscienza di una convinzione contrastante alla condotta pretesa dall'ordinamento giuridico ${ }^{8}$.

Lo studio fenomenologico dell'obiezione di coscienza va a definirne il significato come

un comportamento non violento di chi disobbedisce ad una norma giuridica positiva in base a motivazioni di valore filosofico - non puramente psicologiche - inerente alle personali convinzioni morali, che costituiscono un "dover essere" manifestato dalle norme della propria coscienza e che si oppone al dettato espresso da una norma dell'ordinamento giuridico positivo. Ciò distingue l'obiezione di coscienza dalla trasgressione della legge qualificandola come una forma non arbitraria ed occulta di disobbedienza, dettata da motivi di coscienza e palesata secondo modalità intenzionalmente pubbliche ${ }^{9}$.

${ }^{6}$ Cfr. R. Bertolino, L'obiezione di coscienza moderna. Per una fondazione costituzionale del diritto di obiezione, Torino 1994, p. 1, 28-37.

${ }^{7}$ Cfr. G. Caprarola, Schemi di diritto ecclesiastico, Roma 2014, p. 101.

${ }^{8}$ Cfr. R. Bertolino, op.cit., p. 9.

9 Cfr. V. Turchi, I nuovi volti di Antigone. Le obiezioni di coscienza nell'esperienza giuridica contemporanea, Bologna 2008, p. 11-14. 
L'obiezione di coscienza può intendersi come un conflitto di doveri, di lealtà, di obbedienze tra la coscienza e la norma giuridica. Il termine "obiezione di coscienza" sia contraddistinto da un molteplice significato semantico, da una corrispondenza a due distinti fenomeni che vengono ad essere indicati con uno stesso unico termine. "Per obiezione di coscienza può intendersi, sia nel linguaggio comune sia in quello tecnico-giuridico, o il fatto di qualifica illecita attribuita dall'ordinamento al comportamento dell'obiettore, e allora, più propriamente, l'obiezione di coscienza dovrà qualificarsi come obiezione contraria alla legge". Ultimamente gli ordinamenti democratici che accolgono i valori del pluralismo e del personalismo, riconoscano, a determinate condizioni e nel rispetto di alcuni valori di base, il comportamento dell'obiettore come legittimo. In tal caso l'obiezione di coscienza non viene più considerata illecita, ma come esercizio del diritto. In tale ipotesi l'istanza della coscienza dell'obiettore è ritenuta come meritevole di tutela giuridica e precisamente l'obiezione di coscienza dovrà essere qualificata come obiezione secondo la legge ${ }^{10}$.

L'obiezione di coscienza si concretizza quando all'obbedienza di fronte alla legge viene contrapposta una convinzione di coscienza pienamente inconciliabile, che non permette all'individuo altro che l'inosservanza della norma giuridica. In tal caso è prevista una sanzione, a meno che l'atto di disobbedienza non sia stato legalizzato o giustificato dall'ordinamento, rimanendo impunito in entrambi i casi ${ }^{11}$.

Il diritto all'obiezione di coscienza viene tutelato dal legislatore in seguito al riconoscimento di stati d' animo derivanti dal proprio patrimonio etico-morale e religioso che conduce ad assumere un comportamento ossia ad astenersi dal praticarlo, ancorché vi sia un obbligo giuridico che lo vieti o lo obblighi. Nel rapporto con la società civile quindi l'ordinamento garantisce un equo adattamento con le certezze individuali più intime che possono condurre all'inesigibilità di un comportamento conforme alla legge civile ${ }^{12}$.

L'obiezione di coscienza continua ad avere una fondamentale importanza e una funzione non eliminabile negli ordinamenti giuridici ${ }^{13}$.

\footnotetext{
${ }^{10}$ Ibidem.

${ }^{11}$ Cfr. F. Grandi, Doveri costituzionali e obiezione di coscienza, Napoli 2014, p. 1.

${ }^{12}$ Cfr. F. Velardi, S. Petralia, Diritto ecclesiastico, Roma 2013, p. 161.

${ }^{13}$ Cfr. R. Bertolino, op.cit., p. 13.
} 


\section{OBIEZIONE AL GIURAMENTO}

Nella Costituzione non vi è riferimento al diritto all'obiezione di coscienza, né al principio di libertà di coscienza. Tale circostanza, tuttavia, non ha impedito di dedurre che la Costituzione non abbia riconosciuto l'obiezione di coscien$\mathrm{za}$, ma semplicemente che non si è ritenuto necessario stabilire un'espressa e specifica esenzione dal servizio militare per ragioni di coscienza ${ }^{14}$. Tuttavia la Costituzione non esclude il giuramento come tale, prevedendolo in determinate situazioni (articoli 54, 91 e 93 della Costituzione, nonché art. 5 della legge n. 87 del 11 marzo 1953), ed escludendolo solo nel caso in cui vi sia la possibilità di ledere la libertà di coscienza del giurante ${ }^{15}$.

L'obiezione al giuramento è previsto per alcuni atti processuali, come specificazione del dovere di contribuire alla corretta amministrazione della giustizia, quanto per l'occupazione di pubbliche funzioni, per garantire l'espletamento del dovere di fedeltà alla Repubblica e di osservanza della Costituzione e delle sue leggi. ${ }^{16}$

In tali circostanze, l'obiezione di coscienza può esprimersi mediante differenti modalità: o come rifiuto di giurare secondo la formula religiosa o per opposizione al giuramento in sé.

La problematica del giuramento in ambito processuale può ritenersi in gran parte risolto, sia ad opera del legislatore, per ciò attinente al processo penale, sia in virtù di alcune decisioni della Corte costituzionale in riferimento al processo civile.

\section{SENTENZA N. 58 DEL 6 LUGIO 1960, ORDINANZA N. 15 DEL 17 MARZO 1961, SENTENZA N. 85 DEL 25 MAGGIO 1963}

La giurisprudenza costituzionale maturata sul giuramento durante il processo penale e civile offre la possibilità di concedere un primo spazio alla libertà di coscienza ${ }^{17}$.

${ }^{14}$ Cfr. A. Guarino, Obiezione di coscienza e valori costituzionali, Napoli 1992, p. 5.

${ }^{15}$ Cfr. M. Jasonni, Tra sacro e profano: il tortuoso cammino della Corte costituzionale in tema del giuramento, in R. Botta (a cura di), op.cit., p. 214.

${ }^{16}$ Cfr. A. Guarino, op.cit., p. 77.

${ }^{17}$ Cfr. F. Grandi, op.cit., p. 91. 
La scelta più rilevante proviene dal remoto $1960^{18}$, quando venne sollevata la questione di legittimità costituzionale riguardo l'art. 449 del passato vigente codice di procedura penale che prevedeva al testimone di pronunciare giuramento con formula religiosa. La Corte, dopo aver appurato l'esistenza di un legame necessario "tra l'ammonimento del vincolo religioso e la lettura della formula del giuramento" e che la libertà di pensiero (che nell'ordinanza di rinvio si assumeva a misura costituzionale violata) non poteva essere garantita solo con una riserva mentale del giurante richiamando la giurisprudenza ${ }^{19}$ sul "criterio maggioritario", affermava che la formula utilizzata dalla questione "risponde alla coscienza del popolo italiano, costituito nella quasi totalità di credenti e, quindi, presupponendo nel giurante la credenza in Dio, è adeguata a qualsiasi confessione, anche acattolica" ${ }^{\prime 2}$. Il Giudice costituzionale si pronunciò per l'inconcretezza, asserendo che "il giuramento non impone all'ateo una confessione religiosa" 21 ; ovvero, "a differenza del credente, che è consapevole della responsabilità che col giuramento assume davanti a Dio, l'ateo non viene ad assumere eguale responsabilità verso un Ente Supremo, la cui esistenza egli nega"22. La sentenza in questione non includeva l'ateismo nella libertà religiosa, per il connotato negativo, argomentando a partire dalla libertà di manifestazione del pensiero e quasi a escludere di entrare in un decisione spettante il legislatore. In una successiva ordinanza, n. 15 del $1961^{23}$, venne risollevata la questione di legittimità sull'art. 449 c. p .p. ${ }^{24}$, ma questa volta, i parametri che ritenevano violati erano gli artt. 8 e 19 della Costituzione ${ }^{25}$. La Corte ne rilevò la palese infondatezza, motivando che l'art. 8 non poteva essere posto in relazione "con la pretesa del singolo rivol-

${ }^{18}$ Corte costituzionale, Sentenza 6 luglio 1960, n. 58, in Domianello S., Giurisprudenza costituzionale e fattore religioso. Le pronunzie della Corte costituzionale in materia ecclesiastica (1957-1986), Milano 1987, p. 147-156.

${ }^{19}$ Cfr. Corte costituzionale, Sentenza 28 novembre 1957, n. 125, in Domianello S., Giurisprudenza costituzionale e fattore religioso. Le pronunzie della Corte costituzionale in materia ecclesiastica (1957-1986), Milano 1987, p. 635-642; Sentenza 17 dicembre 1958, n. 79, in Domianello S., Giurisprudenza costituzionale e fattore religioso. Le pronunzie della Corte costituzionale in materia ecclesiastica (1957-1986), Milano 1987, p. 627-630.

${ }^{20}$ Cfr. Corte costituzionale, Sentenza 6 luglio 1960, n. 58, op.cit., p. 153.

${ }^{21}$ Ibidem, p. 154.

${ }^{22}$ Ibidem, p. 154

${ }^{23}$ Corte costituzionale, Ordinanza 17 marzo 1961, n. 15, in Domianello S., Giurisprudenza costituzionale e fattore religioso. Le pronunzie della Corte costituzionale in materia ecclesiastica (1957-1986), Milano 1987, p. 157-160.

${ }^{24}$ Parlamento del Regno d'Italia, Codice di Procedura Penale, Regio Decreto 19 ottobre 1930, n. 1399, "Gazzetta Ufficiale Serie Generale", n. 251 del 26 ottobre 1930.

${ }_{25}$ Cfr. Assemblea Costituente, Costituzione della Repubblica Italiana, "Gazzetta Ufficiale", n. 298 del 27 dicembre 1947. 
ta al riconoscimento del diritto della propria libertà di coscienza e di fede ${ }^{26 "} \mathrm{e}$, quindi riaffermando quanto sostenuto nella precedente sentenza n. 58 del 196027. Qualche anno dopo, la Corte chiamata a pronunciarsi anche sull' art. 251, 2 comma, c. p. c. così disponeva: "Il giudice istruttore ammonisce il testimone sulla importanza religiosa e morale del giuramento e sulle conseguenze penale delle dichiarazioni false o reticenti, e legge la formula - consapevole della responsabilità che con il giuramento assumete davanti a Dio e agli uomini, giurate di dire la verità, null'altro che la verità' ${ }^{28}$.

Mentre in precedenza al n. 85/1963 ${ }^{29}$ la Corte si limitava al caso del cittadino ateo, costretto a dichiarare un Dio non presente nella sua coscienza, essa si pronuncia anche riguardo estende l'ipotesi del cittadino che non intende giurare per rispetto alla propria professione di fede ${ }^{30}$.

\section{SENTENZA N. 117 DEL 2 OTTOBRE 1979}

Riferendosi alla questione del giuramento in sede processuale, la Corte costituzionale, dopo due pronunce contrapposte intervenne con sentenza n. 117 del $1979^{31}$, interpretando un caso di obiezione nel giuramento, cioè quello del testimone che secondo gli artt. 251, 2 comma, codice di procedura civile; inoltre 142, 1 comma; 316, 2 comma; 329, 1 comma e 449, 2 comma, del codice di procedura penale del $1930^{32}$, era chiamato a prestare giuramento con formula religiosa. Per questa motivazione, la sentenza, pur essendo importante per la tutela della libertà di coscienza, lasciava permanere il problema di chi rifiuta il giuramento. A tale riguardo, la Corte, che riveste anche tale ruolo, ritenne espressamente inammissibile la questione proposta, perché ammettere eventuali forme di impe-

${ }^{26}$ Corte costituzionale, Ordinanza 17 marzo 1961, op.cit., p. 159.

${ }^{27}$ Cfr. F. Grandi, op.cit., p. 91-92.

${ }_{28}$ Parlamento del Regno d'Italia, Codice di Procedura Civile, Regio Decreto 28 ottobre 1940, n. 1443, "Gazzetta Ufficiale", n. 253 del 28 ottobre 1940.

${ }^{29}$ Cfr. Corte costituzionale, Sentenza 25 maggio 1963, n. 85, in Domianello S., Giurisprudenza costituzionale e fattore religioso. Le pronunzie della Corte costituzionale in materia ecclesiastica (1957-1986), Milano 1987, p. 161-166.

${ }^{30}$ Cfr. M. Jasonni, op.cit., p. 209.

${ }^{31}$ Corte costituzionale, Sentenza 2 ottobre 1979, n. 117, in Domianello S., Giurisprudenza costituzionale e fattore religioso. Le pronunzie della Corte costituzionale in materia ecclesiastica (1957-1986), Milano 1987, p. 167-178.

32 Parlamento del Regno d'Italia, Codice di Procedura Penale, op.cit. 
gno morale alternative al giuramento avrebbe rappresentato una scelta riservata alla discrezione del legislatore ${ }^{33}$.

Quindi l'incidente di costituzionalità dava origina al rifiuto di giurare di alcuni soggetti - perché atei - chiamati a testimoniare in un processo civile. Gli stessi vennero chiamati a rispondere penalmente per tale posizione tenuta. Nell'ordinanza di rimessione il giudice sosteneva che la coscienza dell'ateo viene forzata "quando questi è costretto a giurare in nome di valori che non riconosce", assumendo, per ciò stesso, che i parametri violati erano gli artt. 2, 3, 19 e 21 della Costituzione. La Corte, ribaltando il precedente indirizzo, dichiarò che la questione era fondata, mettendo in risalto che la formula del giuramento aveva significato religioso, data l'impossibilità a separare le parole pronunciate dal testimone che giura. La novità della sentenza n. 117, tuttavia, deve essere ricercata soprattutto nel fatto che l'ateo subiva un pregiudizio dalla formula recata dalla disposizione impugnata, in ragione dell'art. 19 della Costituzione, che dirige il suo sentimento religioso, o meglio non religioso. Conseguentemente, la Corte precisò che il nocumento alla libertà di coscienza veniva tenuto in considerazione anche nel caso in cui al soggetto fosse stato imposto l'adempimento di atti con significato religioso, tanto che, alla luce della formula recata dall'art. $251 \mathrm{c}$. p. c. e 142 c. p. p. la libertà di coscienza del testimone non credente veniva lesa. La Corte quindi, con una sentenza addizionale, dichiarò illecita la disposizione in questione nella parte in cui non era contenuta l'espressione "se credente", di seguito al riferimento, al significato religioso del giuramento. Ai sensi dell' art 27 della legge n. 87 del $1953^{34}$ la Corte dichiarò l'inconformità costituzionale degli artt. 316 - 2 comma, 329 - 1 comma, 449 - 2 comma e 142 - 1 comma, contenuti nel codice di rito penale recanti le medesime formule. Differentemente, la formula del giuramento decisorio, di cui all'art. 238 c. p. c., non veniva incisa dalla pronuncia in oggetto ${ }^{35}$.

Nonostante le approvazioni per la "svolta", la sentenza n. 117 non sfuggì dalle critiche, il suo testo, non trovava applicazione in tutte le ipotesi di giuramento previste dall'ordinamento, cosicché non si ritenne garantito, a parità di condizioni, lo stesso diritto alla libertà religiosa. Parte della dottrina, ritenne che la Corte avrebbe fatto meglio ad abolire dalla formula del giuramento il riferimento a Dio, piuttosto che andare verso una decisione creativa ${ }^{36}$.

${ }^{33}$ Cfr. V. Turchi, op.cit., p. 112.

${ }^{34}$ Parlamento della Repubblica Italiana, Legge 11 marzo 1953, n. 87, Norme sulla costituzione e sul funzionamento della Corte costituzionale, "Gazzetta Ufficiale Serie Generale", n. 62 del 14 marzo 1953.

${ }^{35}$ Cfr. F. Grandi, op.cit., p. 92-93.

${ }^{36}$ Ibidem, p. 93. 
Con questa sentenza la Corte costituzionale ha modificato la formula del giuramento in ambito processuale ed ha permesso al giurante di impegnarsi solo dinanzi agli uomini o anche dinanzi a Dio, secondo le convinzioni della propria coscienza $^{37}$. "La sentenza n. 117/1979 costituisce una vera svolta, in quanto la Corte riconosce che la formula a contenuto sacrale lede la coscienza del testimone, comprimendo la sua libertà con l'imposizione di assunzione della responsabilità «dinanzi a Dio»"38.

\section{SENTENZA N. 234 DEL 13 LUGLIO 1984, ORDINANZA N. 278 DEL 5 NOVEMBRE 1985}

La sentenza n. 234 del 13 luglio $1984^{39}$ e l'ordinanza n. 278 del 5 novembre $1985^{40}$ relative al giuramento dei testimoni, ove la Corte sulla base delle precedenti pronunce n. 58/60, n. 85/63 e n. 117/79, dichiarano esplicitamente inammissibile per difetto assoluto di motivazione sulla rilevanza la questione di legittimità costituzionale degli artt. 366 c. $\mathrm{p}^{41}$. e 449 c. p. p. nella parte in cui vi è l'obbligo di prestare giuramento anche al testimone la cui religione di appartenenza proibisca di pronunciare le parole "lo giuro" 42.

Inoltre la Corte dichiara prive di riconoscimento le questioni di legittimità costituzionale degli artt. 251 c. p. c., 142 e 449 c. p. p. a motivo della propria impossibilità ad operare "integrazioni e variazioni della normativa in vigore, strettamente dipendenti da una pluralità di scelte discrezionali individuabili dal solo legislatore" ${ }^{43}$.

In tal modo in ambedue i casi si pronunciò a ritenere inammissibile la questione, spiegando che l'eventuale provvedimento di incostituzionalità avrebbe

${ }^{37}$ Cfr. A. Guarino, op.cit., p. 78.

${ }^{38}$ G. Dammacco, op.cit., Napoli 2006, p. 125.

${ }^{39}$ Corte costituzionale, Sentenza 13 luglio 1984, n. 234, in Domianello S., Giurisprudenza costituzionale e fattore religioso. Le pronunzie della Corte costituzionale in materia ecclesiastica (1957-1986), Milano 1987, p. 181-189.

${ }^{40}$ Corte costituzionale, Ordinanza 5 novembre 1985, n. 278, in Domianello S., Giurisprudenza costituzionale e fattore religioso. Le pronunzie della Corte costituzionale in materia ecclesiastica (1957-1986), Milano 1987, p. 190-191.

${ }^{41}$ Parlamento del Regno d'Italia, Codice Penale, Regio Decreto 19 ottobre 1930, n. 1398, "Gazzetta Ufficiale Serie Generale", n. 251 del 26 ottobre 1930.

${ }^{42}$ Cfr. A. Albisetti, Il diritto ecclesiastico nella giurisprudenza della Corte costituzionale, Milano 2010, p. 59.

${ }^{43}$ Ibidem, p. 59-60. 
richiesto degli interventi che sarebbero sconfinati nella discrezionalità politica propria del legislatore ${ }^{44}$.

\section{SENTENZA N. 149 DEL 4-5 MAGGIO 1995}

Nel 1995, la Corte si è pronunciata sulla costituzionalità dell'art. 251 c. p. c., in ragione della differenza di trattamento fra i testimoni nel processo civile e penale, in conseguenza dell'entrata in vigore del nuovo codice di procedura civile (1988), il quale prevedeva all'art. 497, 2 comma, una formula di giuramento per il testimone senza alcun riferimento religioso ${ }^{45}$ :"Consapevole della responsabilità morale giuridica che assumo con la mia deposizione, mi impegno a dire tutta la verità ed a non nascondere nulla di quanto a mia conoscenza"46. Il caso, però, mette in evidenza anche per la diversa prospettazione del dubbio di costituzionalità operata dal giudice ${ }^{47}$.

Anche l'art. 497 del codice di procedura penale del 1988 non prevede più che i testimoni debbano giurare, sono chiamati soltanto ad una esplicita dichiarazione che ha valore di "impegno solenne"

Al fine di assicurare pari tutela al valore della libertà di coscienza riguardo all'obbligo del testimone di impegnarsi a dire la verità, si impone l'estensione dell'art. 251, 2 comma di c. p. c., della disciplina e della formula prevista dell' art. 497, 2 comma di c. p. p., le quali esulano da ogni riferimento a espletazione di giuramento ${ }^{49}$.

Scompare il riferimento alla religione e viene mantenuto l'obbligo morale (oltre che giuridico) di dire la verità ${ }^{50}$.

Le attuali norme sul processo penale (art. 497 c. p. p.) e sul processo civile (art. 251 c. p. c.) danno forma al giuramento dei testimoni non come un'assunzione di responsabilità riferita a Dio, se credente, o agli uomini, ma obbligano il giudice di rendere chiaro al testimone l'obbligo di dire la verità e le conseguenze penali relative a dichiarazioni false e reticenti e la dichiarazione del testimone

${ }^{44}$ Cfr. A. Guarino, op.cit., p. 93-94.

${ }^{45}$ Corte costituzionale, Sentenza 4-5 maggio 1995, n. 149, in Domianello S., Giurisprudenza costituzionale e fattore religioso. Le pronunzie della Corte costituzionale in materia ecclesiastica (1987-1998), Milano 1999, p. 307-316.

${ }^{46}$ Presidente della Repubblica, Codice di Procedura Penale, Decreto 22 settembre 1988, n. 447, "Gazzetta Ufficiale", n. 250 del 24 ottobre 1988 - Suppl. Ordinario n. 92.

${ }^{47}$ Cfr. F. Grandi, op.cit., p. 94.

${ }^{48}$ Cfr. A. Albisetti, op.cit., p. 60.

${ }^{49}$ Ibidem, p. 95

${ }^{50}$ Ibidem. 
nella forma e struttura si configura come impegno a dire tutta la verità e a non nascondere nulla di quanto conosce nella consapevolezza della responsabilità morale e giuridica che si assume con la deposizione ${ }^{51}$.

Soltanto con l'entrata in vigore dell'ultimo codice di procedura penale vi è stato un intervento esplicito del legislatore, mentre per adeguare la corrispondente norma processuale civile sono stati necessari due interventi della Corte costituzionale (sentenza n. 117 del 1979 e sentenza n. 149 del 1995).

L'occasione dell'incidente di costituzionalità è avvenuto in concomitanza del rifiuto manifestato da alcuni soggetti di rendere testimonianza davanti ai loro convincimenti religiosi. Il giudice rimettente poté chiedere alla Corte di pronunciarsi sull'obiezione di coscienza all'ufficio testimoniale anche per i "testimoni credenti che per motivi di fede o in adesione ai principii della propria confessione religiosa, ricusino di prestare giuramento con qualunque formula ovvero con una formula, come quella attuale nel processo civile, contenente un esplicito riferimento alla divinità" 52 .

Anche in questa occasione non si trovò la soluzione al problema relativo alla formula del giuramento decisorio, sul quale il Giudice costituzionale tornò l'anno successivo ${ }^{53}$. In particolare, la lettera dell' art. 238 c. p. c. così stabiliva: “[... il giudice] ammonisce il giurante sull'importanza religiosa e morale dell'atto e sulle conseguenze penali delle dichiarazioni false, e quindi lo invita a giurare [...]: «consapevole della responsabilità che col giuramento assumo davanti a Dio e agli uomini giuro» [...]"54. La questione di costituzionalità rilevò che la diversità delle formule sacramentali violava il principio di eguaglianza. La Corte accolse la discussione dell' argomento esclusivamente in relazione al contrasto della disposizione con la libertà religiosa e non - al pari della sentenza del 1995 - a sostituzione della formula in ragione della diversità degli istituti a confronto $^{55}$.

Con la stessa sentenza la Corte costituzionale ha dichiarato l'illegittimità costituzionale dell' art. 251, 2 comma, del codice di procedura civile in relazione agli articoli 3 e 19 della Costituzione ${ }^{56}$.

${ }^{51}$ Cfr. Presidente della Repubblica, Codice di Procedura Penale, op.cit.; Parlamento del Regno d'Italia, Codice di Procedura Civile, op.cit.

${ }^{52}$ Corte costituzionale, Sentenza 4-5 maggio 1995, n. 149, op.cit., p. 309.

${ }^{53}$ Cfr. F. Grandi, op.cit., p. 94.

${ }_{54}$ Parlamento del Regno d'Italia, Codice di Procedura Civile, op.cit.

${ }_{55}$ Cfr. F. Grandi, op.cit., p. 94.

${ }^{56}$ Cfr. Parlamento del Regno d'Italia, Codice di Procedura Civile, op.cit. Assemblea Costituente, Costituzione della Repubblica Italiana, op.cit. 


\section{SENTENZA N. 334 DEL 8 OTTOBRE 1996}

La Corte costituzionale afferma che "gli artt. 2, 3 e 19 Cost. garantiscono come diritto la libertà di coscienza nella sfera religiosa. Tale diritto, nel profilo giuridico-costituzionale, rappresenta uno degli aspetti legati alla dignità della persona riconosciuta e dichiarata inviolabile dall' art. 2. Spetta in egual misura ai credenti e ai non credenti, siano essi atei o agnostici (sentenza n. 117 del 1979) e determina la conseguenza, valida nei confronti degli uni e degli altri, che in nessun caso il compimento di atti appartenenti alla sfera religiosa possa essere oggetto di obblighi derivanti dall'ordinamento giuridico statale ${ }^{57}$.

Trattasi dunque non soltanto della protezione coscienza dei non credenti che non possono essere obbligati a pronunciarsi con atti il cui significato contrasti con le loro convinzioni, viene ad essere toccata la natura stessa dell'essere religioso che, nell'ordine civile, per l'ordinamento costituzionale può essere considerata solo manifestazione di libertà. Qualunque atto che abbia significato religioso, fosse pure il più doveroso rispetto all'ortodossia di una religione e delle sue istituzioni, rappresenta sempre per lo Stato un esercizio della libertà dei propri cittadini, manifestazione che come non può essere tradotta con una prescrizione obbligatoria, indipendentemente dall'irrilevante circostanza che il suo contenuto sia conforme, estraneo o contrastante con la alla coscienza religiosa individuale $^{58}$.

La Corte non si limita ad eliminare il riferimento alla divinità presente nella originaria formula del giuramento ex art. 238, 2 comma del codice di procedura civile, ma dichiara costituzionalmente illegittimo anche il riferimento alla responsabilità che il dichiarante assume davanti agli uomini. Così nella discussione i giudici della Consulta: per un verso dalla dichiarazione d'incostituzionalità dei soli riferimenti alla divinità potrebbe apparire legittimata una sorta di religione dell'umanità e per altro verso reso palese il riferimento a un solo contenuto di valore, vengono esclusi gli altri, con violazione della libertà di coscienza dei credenti, per i quali il giuramento ha un significato religioso ${ }^{59}$.

In considerazione di ciò per lo Stato il divieto di fare ricorso a obbligazioni di ordine religioso per giungere a consolidare l'efficacia dei propri precetti ${ }^{60}$. Particolarmente, il richiamo del giudice sull'importanza religiosa del giuramen-

${ }^{57}$ Cfr. Corte costituzionale, Sentenza 8 ottobre 1996, n. 334, in Domianello S. (1999), Giurisprudenza costituzionale e fattore religioso. Le pronunzie della Corte costituzionale in materia ecclesiastica (1987-1998), Milano, p. 320.

${ }^{58}$ Ibidem, p. 320-321.

${ }^{59}$ Ibidem, p. 326.

${ }^{60}$ Ibidem, p. 321. 
to (art. 238, 1 comma del codice di procedura civile), nonché la formula religiosa del giuramento stesso (art. 238, 2 comma del codice di procedura penale) vengono a delineare "un'inammissibile commistione: un'obbligazione di natura religiosa e il vincolo che ne deriva nel relativo ambito sono imposti per un fine probatorio proprio dell'ordinamento processuale dello Stato" ${ }^{\text {. }}$.

La sentenza n. 334 del 1996 dichiara che la libertà di coscienza unisce credenti e non, la libertà di coscienza rappresenta una garanzia per i laici, ed anche forse, per i credenti. La libertà di coscienza consente a questi ultimi di fare opposizione alla legge, in quanto cittadini, appellandosi al diritto sancito nell'obiezione di coscienza rispetto ad un precetto che appare in contrasto con la loro fede e in quanto credenti, conferisce agli stessi la facoltà di non ottemperare ai comandi del magistero confessionale in contrasto con la loro coscienza, vista la non precettività di detti comandi ${ }^{62}$.

La Corte costituzionale mette in evidenza che la libertà di coscienza non viene garantita quando ad un non credente vengono richiesti atti con significato religioso. Lo scombussolamento di coscienza può interessare coloro la cui religione di appartenenza faccia divieto di prestare giuramento ${ }^{63}$. Alla stessa soluzione la Corte è giunta anche per il giuramento decisorio previsto dal codice di procedura civile (sentenza n. 334 del 1996), che oggi viene pronunciato con la formula: «consapevole della responsabilità che col giuramento assumo ... giuro.... $»^{64}$.

Con questa decisione sono state escluse dal diritto processuale tutte le forme di giuramento, ritenendo soddisfatte le esigenze scaturite nei casi di obiezione di coscienza. Questa decisione non è ancora soddisfatta per i numerosi casi di giuramento previsti in ambito amministrativo o nello stesso ambito giudiziario, come nel caso di assunzione di particolari incarichi o funzioni ${ }^{65}$.

\section{CONCLUSIONI}

La giurisprudenza della Corte costituzionale relativa all'obiezione di coscienza al giuramento ha risolto la questione ed il problema di coloro che per

${ }^{61}$ Ibidem, p. 322.

${ }^{62}$ Cfr. F. Grandi, op.cit., p. 97.

${ }^{63}$ Cfr. F. Del Giudice, Compendio di diritto ecclesiastico. Chiese, culti e religioni nell'ordinamento italiano, Napoli 2014, p. 202.

${ }^{64}$ Corte costituzionale, Sentenza 8 ottobre 1996, n. 334, op.cit. p. 318.

${ }^{65}$ Cfr. L. Musselli, C. B. Ceffa, Libertà religiosa, obiezione di coscienza e giurisprudenza costituzionale, Torino 2014, p. 45. 
motivi di coscienza non vogliono giurare nel processo penale o civile poiché nelle formule codiciali erano presenti riferimenti di carattere religioso, in seguito aggiornate con le suddette sentenze ed approvate nei codici dal legislatore.

Le norme dei giuramenti relativi a Dio e a riferimenti religiosi, modificate dall'obbligo di dire la verità, hanno fatto sì che le persone coinvolte nei processi non si trovassero più ad affrontare il problema dell'obiezione di coscienza dato che sono stati omessi i valori e terminologia che possono sottolineare i dati personali in termini di affiliazione religiosa o di altro genere.

La medesima Corte ha dichiarato che compete al legislatore decidere in quale modo rafforzare il significato del giuramento con un appello rivolto a chi è tenuto a prestarlo affinché faccia appello ai valori che più onora nell'intimo della sua coscienza, quelli religiosi ed etici; fermo restando rimanga illesa la libertà di coscienza di coloro che devono giurare ${ }^{66}$.

\section{BIBLIOGRAFIA:}

Albisetti A. (2010), Il diritto ecclesiastico nella giurisprudenza della Corte costituziona$l e$, Giuffrè Editore, Milano.

Assemblea Costituente, Costituzione della Repubblica Italiana, "Gazzetta Ufficiale", n. 298 del 27 dicembre 1947.

Bertolino R. (1994), L'obiezione di coscienza moderna. Per una fondazione costituzionale del diritto di obiezione, G. Giappichelli Editore, Torino.

Caprarola G. (2014), Schemi di diritto ecclesiastico, Nel Diritto Editore, Roma.

Dammacco G. (2006), L'obiezione di coscienza nella giurisprudenza della Corte costituzionale, in Botta R. (a cura di), Diritto ecclesiastico e Corte costituzionale, Edizioni Scientifiche Italiane, Napoli, p. 113-135.

Del Giudice F. (2014), Compendio di diritto ecclesiastico. Chiese, culti e religioni nell'ordinamento italiano, Edizioni Giuridiche Simone, Napoli.

Grandi F. (2014), Doveri costituzionali e obiezione di coscienza, Editoriale Scientifica, Napoli

Guarino A. (1992), Obiezione di coscienza e valori costituzionali, Jovene Editore, Napoli.

Jasonni M. (2006), Tra sacro e profano: il tortuoso cammino della Corte costituzionale in tema del giuramento, in Botta R. (a cura di), Diritto ecclesiastico e Corte costituzionale, Edizioni Scientifiche Italiane, Napoli, p. 207-215.

${ }^{66}$ Cfr. Corte costituzionale, Sentenza 2 ottobre 1979, n. 117, op.cit., p. 176. 
Musselli L., Ceffa C. B. (2014), Libertà religiosa, obiezione di coscienza e giurisprudenza costituzionale, G. Giappichelli Editore, Torino.

Skwarczyński M, Sprzeciw sumienia w europejskim i krajowym systemie ochrony praw człowieka, „Przegląd Sejmowy”, 6 (119)/2013, p. 9-26.

Turchi V. (2008), I nuovi volti di Antigone. Le obiezioni di coscienza nell'esperienza giuridica contemporanea, D. U. Press, Bologna.

Velardi F., Petralia S. (2013), Diritto ecclesiastico, Nel Diritto Editore, Roma.

Zoll A. (2014), Klauzula sumienia, in Stanisz P., Pawlikowski J., Ordon M. (a cura di), Sprzeciw sumienia $w$ praktyce medycznej - aspekty etyczne $i$ prawne, Wydawnictwo KUL, Lublin, p. 77-86. 
